\title{
Repeated isolation experience during the preweanling period: Effects on behavioral ontogeny
}

\author{
PATRICIA A. CAZA, W. DOUGLAS TYNAN, GAYLE SOLHEIM, \\ and NORMAN E. SPEAR \\ Department of Psychology and Center for Neurobehavioral Sciences \\ State University of New York, Binghamton, New York 13901
}

\begin{abstract}
Two experiments investigated the effects of repeated isolation of rat pups from the dam and conspecifics for $3 \mathrm{~h}$ daily commencing on the day following parturition and continuing until Postnatal Day 21. Sensorimotor development, nest-directed behavior (homing), and general activity were assessed during these 21 days. Results indicated that pups experiencing repeated isolation exhibited an earlier mature tail-hang and olfactory orientation response, somewhat more nest-directed behavior, and greater activity in an open field. These effects could be attenuated by isolating the rats with some aspect of the home nest environment, such as conspecifics or home nest litter.
\end{abstract}

Numerous investigations have been conducted on the impact of early life experiences, such as hormonal intervention, malnutrition, environmental enrichment, and handling, on later behavior in the rodent. Henderson (1980) has stated that the area of slowest progress in understanding the behavioral and physiological consequences of early life experiences is in the effects of early handling or stress. This may, in part, be due to the nature of this type of experimental treatment, which has ranged from short "gentling" periods to chronic total isolation of rat pups from their dam and conspecifics. In addition, the majority of studies in this area have waited until subjects reached adulthood to examine their behavior following early treatment experiences. A more sensitive tool for assessing effects of these early experiences could be the relative rates of development in treated vs. untreated organisms. Because the mammalian brain has remarkable compensatory mechanisms for dealing with early alterations in its normal course of development, an examination of ontogenetic differences in rat pups subjected to early treatment experiences may prove more fruitful.

Intuitively, one of the most stressful early life experiences for any young altricial organism would be isolation from the home environment. Thoman and Arnold (1968) reported that adult rats that had been totally isolated during their preweanling period displayed superior

This research was supported by grants from the National Science Foundation (BNS74-24194 and BNS78-02360) and the National Institute of Mental Health (1R01 MH35219-01) to Norman E. Spear. The authors wish to express their appreciation to Norman G. Richter, Sr., and Teri Tanenhaus. Some of these data were also presented at the 51st annual meetings of the Eastern Psychological Association, Hartford, Connecticut, 1980. Requests for reprints should be addressed to Norman E. Spear, Department of Psychology, Binghamton, New York 13901.
Hebb-Williams maze performance and a greater change over days in their open-field behavior relative to normally reared rats. Because of the difficulty in maintaining pups away from the home nest for the entire preweanling period, most research investigating isolation effects on behavior has employed isolation treatments that are initiated following weaning (e.g., Katz \& Steinberg, 1970; Valzelli, 1973; Weinstock, Speiser, \& Ashkenazi, 1978).

The present experiments attempted to discern what effects repeated bouts of acute isolation experience, commencing on the day following parturition, would have on the rate of behavioral ontogeny. In addition to singly isolating pups, some subjects were isolated with two conspecifics. The behavioral consequences of various early life treatments have been reported to be less in group-reared or group-tested animals (e.g., Pearson, Teicher, Shaywitz, Cohen, Young, \& Anderson, 1980; Randall \& Campbell, 1976).

\section{EXPERIMENT 1}

\section{Method}

Subjects. Fifty-three Sprague-Dawley-derived experimentally naive albino rat pups of both sexes from six litters (litter size $=$ 8-10) were used. Pups were housed with both parents and maintained on a 16-h light/8 h dark illumination cycle, with light onset at $1800 \mathrm{~h}$. Date of birth is considered to be Day 0 postpartum.

Apparatus. Isolation chambers consisted of opaque maternity cages partially filled with clean pine shavings. Chambers for the I subjects measured $22.9 \times 11.4 \times 15.2 \mathrm{~cm}$; chambers for the IC subjects measured $25.4 \times 14.0 \times 12.7 \mathrm{~cm}$. Heating pads were placed under all isolation chambers to maintain temperatures at $33^{\circ} \pm 1^{\circ} \mathrm{C}$. Isolation and testing were conducted during the subjects' dark cycle; red-light illumination was used.

The homing apparatus consisted of a five-sectioned $38.0 \mathrm{x}$ $19.0 \times 10.1 \mathrm{~cm}$ Plexiglas box, covered with a fine wire-mesh screen marked into $3.3 \times 3.8 \mathrm{~cm}$ squares. Clean pine shavings 
were placed under four of the adjoining sections, and under the fifth section, 7-day-old home nest shavings were placed.

The open-field chamber was a $30.5 \times 30.5 \times 30.5 \mathrm{~cm}$ glass box; the floor was marked into $7.6 \times 7.6 \mathrm{~cm}$ squares.

Procedure. Pups within each litter were randomly assigned to one of three experimental conditions on Day 1: individual isolation (I), isolation with two conspecifics (IC), and control, left with the parents (MC). Within each litter, at least two pups were assigned to the MC condition, three pups to the IC condition, and the remainder (maximum of four pups) to the I condition. There were 20 pups in the I condition, 17 in the IC condition, and 16 in the MC condition.

Isolation treatment was conducted for $3 \mathrm{~h}$ daily from Day 1 to Day 21. Pups in the I group were placed singly into the isolation boxes, and IC-treated pups were placed three to an isolation chamber. MC subjects were left with their parents in the home cage in the vivarium throughout the isolation period, except when they were transported to the experimental room for testing. Each day, all pups were color coded with felt markers and weighed; thus, all subjects were handled to some degree. Testing on any given day began approximately $15 \mathrm{~min}$ after the isolation period began.

Starting on Day 1, all pups were weighed and tested for tailhang response using the procedure employed by Almli and Fisher (1977). The appearance of this response was assessed daily until a subject exhibited a mature response for 2 consecutive days (moving the head in both directions and twisting the torso upward). Daily weighing continued until Day 15 .

On Day 7, each pup was given a homing test. The test began by orienting the pup $90 \mathrm{deg}$ from the location of the home shavings in the middle section of the apparatus. The number of squares crossed in each of the five sections and the latency to reach the "home" area were recorded during the 180 -sec test.

On Day 21, a 3-min open-field activity test was given. Subjects were individually tested, and the number of squares crossed was recorded.

\section{Results}

All dependent measures were analyzed using unweighted-means analysis of variance (ANOVA) techniques. Multiple comparisons using Duncan's test were also employed $(\mathrm{p}<.05)$.

On Day 1, all pups were equivalent in weight (initial weight: $\overline{\mathrm{X}}_{\mathrm{I}}=7.6 \mathrm{~g}, \overline{\mathrm{X}}_{\mathrm{IC}}=7.7 \mathrm{~g}, \overline{\mathrm{X}}_{\mathrm{MC}}=7.3 \mathrm{~g}$ ) and maintained this equivalence across days $(F<1)$. The age of reaching criterion for the tail-hang response was significantly younger for the I subjects (Day 6.5) than for either the IC or MC subjects $\left[\overline{\mathrm{X}}_{\mathrm{IC}}=8.65, \overline{\mathrm{X}}_{\mathrm{MC}}=9.25\right.$; $\mathrm{F}(2,50)=6.99, \mathrm{p}<.05]$.

Performance on the homing task also differed among the three conditions; $75 \%$ of the I subjects reached home within the 180 -sec criterion, whereas only $53 \%$ of the IC subjects and only $66 \%$ of the MC subjects did so. In addition, MC subjects crossed fewer squares in the homing apparatus than did I or IC subjects $[\mathrm{F}(2,50)=11.74$, $\mathrm{p}<.01]$. Subjects in the I condition were somewhat faster in reaching the "home" area than were those in the IC condition; $\mathrm{MC}$ subjects were intermediate to these extremes $[F(2,50)=2.84, \mathrm{p}<.06]$. Figure 1 illustrates the latency and activity scores for the three groups in the homing task. The seemingly anomalous independence between activity and latency scores for the IC group on Postnatal Day 7 can be explained by examining relative activity for each of the five partitions. Pups in
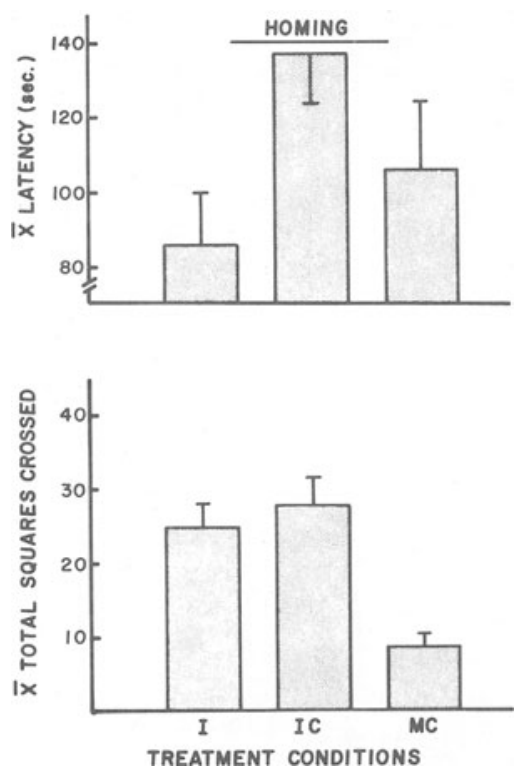

Figure 1. Homing performance of isolate (I), isolate control (IC), and mothered control (MC) subjects on Postnatal Day 7. Upper half represents mean latency to reach home nest area; lower half represents mean number of squares crossed during 3-min test. Lines above bars represent standard errors of the mean.

the IC condition were much more active in the starting area and less active in the home area than were I pups, thus equalizing the total activity of the two groups (start area: $\overline{\mathrm{X}}_{\mathrm{I}}=6.15, \overline{\mathrm{X}}_{\mathrm{IC}}=10.6$; home area: $\overline{\mathrm{X}}_{\mathrm{I}}=10.1$, $\mathrm{X}_{\mathrm{IC}}=5.2$ ).

On Day 21, I subjects also crossed more squares during the 3-min open-field test than did subjects in the other two groups $\left[\overline{\mathrm{X}}_{\mathrm{I}}=84.8, \overline{\mathrm{X}}_{\mathrm{IC}}=68.7, \overline{\mathrm{X}}_{\mathrm{MC}}=69.4\right.$; $\mathrm{F}(2,50)=4.51, \mathrm{p}<.05]$.

\section{Discussion}

The earlier emergence of an adult tail-hang response in the individually isolated rat pups suggests that $3 \mathrm{~h}$ of isolation stress daily during the first 5 days of life can facilitate development of at least one sensorimotor capability. Almli and Fisher (1977) reported that this response is displayed in normal untreated pups at approximately Day 10 , with roughly $50 \%$ of the pups displaying this response at Day 6. In this experiment, pups in the I condition displayed a mature response at Day 6.5; this becomes a somewhat startling facilitation when it is noted that the criterion for a mature response employed in the present study was more stringent than that of Almli and Fisher. Pups in this experiment had to display the adult-like behavior for 2 consecutive days, whereas Almli and Fisher used a 1-day criterion.

Like most behaviors, homing has been considered a composite of sensory, motivational, and motoric functions (Altman, Sudarshan, Das, McCormick, \& Barnes, 1971). The faster latency to home exhibited by I subjects, along with their relatively higher probability for successfully homing, suggests that perhaps these pups (I) had a greater "motivation" to be back in the nest than did the IC or MC pups. The faster latency to home for the I subjects could also reflect a greater motoric development in these pups relative to the other two groups. This would agree with the advanced sensorimotor development of the tail-hang response observed in these I subjects. However, general activity may also be considered to reflect motoric development, and I and IC subjects were equally active in the homing task. It may 
be, therefore, that the superior overall homing performance of I subjects in this experiment reflects a motivational difference in addition to a more advanced motoric response.

The increased open-field activity in the I subjects relative to IC and MC subjects at Day 21 is in agreement with a majority of the literature, and it can be interpreted in a variety of ways, for example, as due to a smaller degree of emotional reactivity to novel situations among I compared to IC and MC subjects (for reviews of the effects of early life experiences, see Daly, 1973; Henderson, 1980; Russell, 1971).

The major findings of this study, the earlier emergence of the mature tail-hang response, the superior homing performance, and the greater activity in I-treated subjects, suggest that isolation stress for $3 \mathrm{~h}$ daily can alter the rate of development of young rat pups.

\section{EXPERIMENT 2}

Numerous studies have shown a facilitation effect of home nest shavings or nest odors on behavior in developing rats (e.g., Smith \& Spear, 1978). It is possible that if home nest shavings were present during isolation, the effects of chronic daily isolation would be mitigated due to the increased similarity of the isolation chamber and the home nest. To test this possibility, four groups of pups received either the I or IC treatments in the context of either home nest or clean shavings.

\section{Method}

Subjects and Apparatus. The subjects were 49 rat pups from five litters, housed like those in Experiment 1. The isolation chambers for the I and IC conditions, the homing apparatus, and the open-field box were the same as those used in Experiment 1.

Procedure. Pups from each litter were randomly assigned to either the I or IC treatment conditions. As in Experiment 1, the pups daily were placed individually (I) or in groups of three (IC) into the isolation chambers for a 3-h period. For half the subjects in each condition, the chambers were partially filled with clean shavings, and for the other half, the chambers contained shavings that were taken from the pups' home nest. Thus, there were four experimental conditions: IC/home shavings (IC-HS), $\mathrm{n}=15 ; \mathrm{IC} /$ clean shavings (IC-CS), $\mathrm{n}=15 ; \mathrm{I} /$ home shavings (I-HS), $\mathrm{n}=9$; and $\mathrm{I} /$ clean shavings (I-CS), $\mathrm{n}=10$.

Pups were isolated from Day 1 to Day 21 and weighed on Days 1-7. Starting on Day 1, all pups were tested for the tailhang response, as in Experiment 1. They were also tested for rooting and olfactory orientation, using the procedures employed by Almli and Fischer (1977). These responses were assessed daily until a subject exhibited a mature response for 2 consecutive days. For olfactory orientation, two cotton-tipped applicators, one free of odor and one that had been dipped into either milk or methyl salicylate, were held in a V shape at the snout level. A response could either be a movement toward the stimulus or a movement toward the stimulus followed by a withdrawal. The order of daily presentation of either odor to each animal was counterbalanced across days. The rooting response was defined as an upward pressure by the head of the rat pups when the experimenter's thumb and index finger were placed over the dorsal surface of the snout and head (Almli \& Fischer, 1977).

A homing test was conducted on Day 7 and open-field activity was assessed on Day 21 using the procedures outlined in Experiment 1. On Days 7 and 14, home nest boxes were replaced with clean shavings after isolation and testing.

\section{Results and Discussion}

Unless otherwise noted, all analyses were 2 (isolation) by 2 (shavings) ANOVAs and multiple comparisons using Duncan's test.
Maturation of the rooting response did not differ among the four treatment conditions ( $\mathrm{Fs}<1$; overall mean $=4.1$ days). Almli and Fischer (1977) reported that their subjects exhibited the mature rooting response between Day 1 and Day 6.

Isolation in clean shavings facilitated maturation of the tail-hang response (Day 6.6) relative to those isolated in home nest shavings (Day 8.6) for I and IC subjects $[F(1,44)=12.36, p<.005]$. There was, however, a mild discrepancy in the mean criterion day for the I-CS and IC-CS groups compared with those in Experiment 1, apparently due to a faster maturation of the tail-hang response in IC subjects in the present experiment (Experiment 1: $\quad \overline{\mathrm{X}}_{\mathrm{I}}=6.5, \quad \overline{\mathrm{X}}_{\mathrm{IC}}=8.65 ;$ Experiment 2: $\overline{\mathrm{X}}_{\mathrm{I}-\mathrm{CS}}=6.11, \overline{\mathrm{X}}_{\mathrm{IC}-\mathrm{CS}}=6.9$ ). The development of olfactory orientation was also influenced by the type of shavings present during the isolation treatment but not by the type of treatment itself. I and IC subjects did not differ in the age at which they achieved the orientation criterion $(F<1)$, but those pups isolated in home nest shavings showed the olfactory orientation approximately 1 day later (Day 8.4) than those isolated in clean shavings (Day 7.3) $[F(1,44)=7.29, p<.05]$.

The day of emergence of these responses for subjects isolated in their home nest shavings closely corresponds to the developmental data reported by Almli and Fischer (1977). In their study, subjects were removed from the nest only for testing. It seems, therefore, that the presence of home nest shavings is sufficient to assure normal development of both a tail-hang response and olfactory orientation, even in the temporary absence of the parents.

Performance on the homing task did not differ among the four treatment conditions with respect to either latency or total activity measures $(\mathrm{Fs}<1)$. The percentage of pups that reached the home area within the 180 -sec criterion also did not differ [I-HS $=100 \%$, I-CS $=78 \%$, IC-HS $=80 \%$, IC-CS $=80 \% ; \chi^{2}(3)=3.04$, $\mathrm{p}>.05]$. This failure to replicate the latency differences reported in Experiment 1 can be attributed to a ceiling effect on measurement in the present experiment caused by the larger percentage of IC-CS pups reaching the home in this experiment relative to Experiment 1 (80\% vs. $53 \%$ ).

As in Experiment 1, I subjects were more active in the open field than IC subjects $[F(1,44)=8.28, p<.01]$. However, this difference was somewhat dependent upon the type of shaving present during the isolation period [Isolation by Shavings interaction, $\mathrm{F}(1,44)=2.92$, $\mathrm{p}<.10]$. Figure 2 illustrates that I-CS subjects crossed more squares than subjects in the other three groups, which did not differ from each other $(p<.05$, Duncan's multiple comparison test). This equivalence in activity level between the two IC groups and the I-HS group suggests that the presence of some aspect of the home nest environment during isolation can attenuate the hyperactivity induced on Day 21 by chronic daily isolation treatment. That the IC-CS and IC-HS subjects crossed an equal number of squares in the open field 


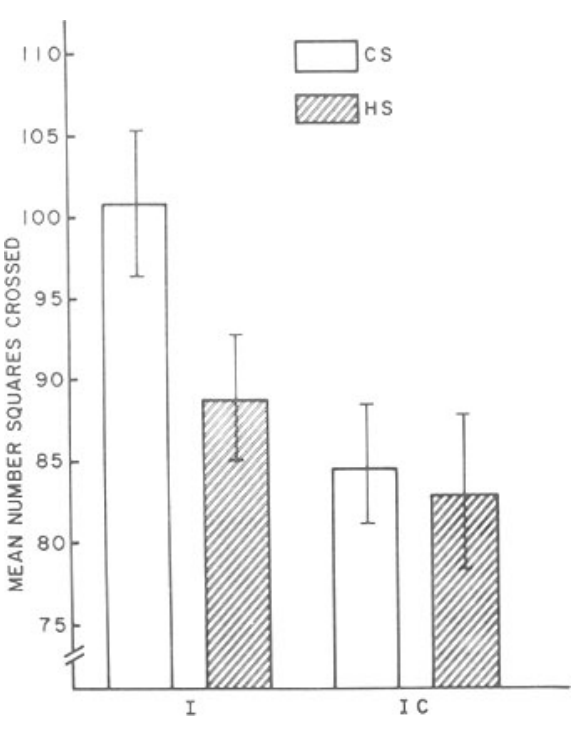

Figure 2. Mean number of squares crossed during open-field test for isolate (I) and isolate control (IC) subjects isolated in either clean (CS) or home nest (HS) shavings. Lines above bars represent standard errors of the mean.

implies that the effects of conspecifics and home shavings present during the isolation period are not additive (i.e., each treatment alone produced the maximal alleviation of the isolation-induced hyperactivity).

The present experiment demonstrated that the presence of home nest shavings during the isolation period can attenuate several effects of isolation, the faster maturation of the tail-hang and olfactory orientation responses observed in I-CS and IC-CS pups, and the increased activity in the open-field test seen in the I-CS subjects.

\section{GENERAL DISCUSSION}

These two experiments illustrate that chronic daily isolation from the nest and conspecifics can facilitate sensorimotor development (i.e., tail hang and olfactory orientation) and perhaps performance in returning to the nest in a homing task. This chronic isolation can produce hyperactivity as assessed in an open-field test. Thus, not only does chronic early isolation treatment alter the behavioral responses of rats in later life (e.g., Thoman \& Arnold, 1969), but it also alters the development of certain behaviors in early life.

The results of Experiment 2 indicate that the presence of either home nest material or conspecifics can attenuate the effects of chronic early isolation. Campbell and Raskin (1978) have also reported that the hyperactivity observed in 15-day-old rats can be decreased if the pups are tested in the presence of home nest material. Perhaps these home nest cues (i.e., shavings, conspecifics) decrease the stress associated with the chronic isolation experience. Campbell and Raskin have proposed a similar model to explain why the presence of conspecifics can depress the hyperactivity seen in 15-day-old rats. They propose that the 15-day-old rat is uniquely sensitive to the dissimilarity between the testing and the home environment and that this sensitivity is manifested by hyperactivity in the testing environment. Smith and Spear (1980) have similarly suggested that the facilitated learning abilities of rat pups trained with aspects of the home nest environment present may be related to the pups' ability with these cues to better "channel arousal levels into more appropriate behaviors which are compatible with the learning situation" (p. 494). Before, however, any conclusions can be drawn concerning the importance of stress for producing the various effects observed following chronic isolation and the attenuation of this stress response by home nest-related cues, independent physiological measures of stress, such as corticosterone levels, must be assessed.

\section{REFERENCES}

Almli, D. R., \& Fisher, R. S. Infant rats: Sensorimotor ontogeny and effects of substantia nigra destruction. Brain Research Bulletin, 1977, 2, 425-429.

Altman, J., \& Sudarshan, K. Postnatal development of locomotion in the laboratory rat. Animal Behavior, 1975, 23, 896920.

Altman, J., Sudarshan, K., Das, G. D., McCormick, N., \& BARNES, D. The influence of nutrition on neural and behavioral development. III. Development of some motor, particularly locomotor patterns. Developmental Psychobiology, 1971, 4, 97-114.

Campbell, B. A., \& Raskin, L. A. Ontogeny of behavioral arousal: The role of environmental stimuli. Journal of Comparative and Physiological Psychology, 1978, 92, 176-184.

DALY, M. Early stimulation of rodents: A critical review of present interpretations. British Journal of Psychology, 1973, 64, 435-460.

HENDERSON, N. D. Effects of early experience upon the behavior of animals: The second twenty-five years of research. In E. C. Simmel (Ed.), Early experiences and early behavior. New York: Academic Press, 1980.

Katz, D. M., \& Steinberg, H. Long-term isolation in rats reduces morphine response. Nature, 1970, 228, 469-471.

Pearson, D. E., Teicher, M. H., Shaywitz, B. A., Cohen, D. J., Young, J. G., \& Anderson, G. M. Environmental influences in body weight and behavior in developing rats after neonatal 6-hydroxydopamine. Science, 1980, 209, 715717.

RANDAll, P. K., \& CAMpbell, B. A. Ontogeny of behavioral arousal in rats: Effects of maternal and sibling presence. Journal of Comparative and Physiological Psychology, 1976, 90, 453-459.

Russell, P. A. Infantile stimulation in rodents. Psychological Bulletin, 1971, 75, 192-202.

Smith, G. J., \& Spear, N. E. Effects of the home environment on withholding behaviors and conditioning in infant and neonatal rats. Science, 1978, 202, 327-329.

Smith, G. J., \& SPEAR, N. E. Facilitation of conditioning in two-day old rats by training in the presence of conspecifics. Behavioral and Neural Biology, 1980, 28, 491-495.

Thoman, E. B., \& ARnOLD, W. J. Effects of incubator rearing with deprivation on maternal behavior in rats. Journal of Comparative and Physiological Psychology, 1968, 65, 441-446.

VAlzelli, L. The "isolation syndrome" in mice. Psychopharmacology, 1973, 31, 305-320.

Weinstock, M., Speiser, Z., \& Ashkenazi, R. Changes in brain catecholamine turnover and receptor sensitivity induced by social deprivation in rats. Psychopharmacology, 1978, 56, 205-209.

(Received for publication June 11, 1982.) 